\title{
High Daytime and Nighttime Temperatures Exert Large and Opposing Impacts on Winter Wheat Yield in China
}

\author{
Xiaoguang Chen, Guoping Tian, And Zhilong Qin \\ Research Institute of Economics and Management, Southwestern University of Finance and Economics, \\ Chengdu, China \\ XIANG BI \\ Department of Food and Resource Economics, University of Florida, Gainesville, Florida
}

(Manuscript received 11 February 2019, in final form 22 June 2019)

\begin{abstract}
We analyze a provincial-scale dataset of winter wheat yield, together with finescale daily weather outcomes from 1979 to 2011, to assess the responses of winter wheat yield in China to temperature fluctuations. Contrary to the majority of the previous literature, we find that winter wheat yield in China responded positively to higher nighttime temperature $T_{\min }$, with the positive $T_{\min }$ effects most significant in the northern China winter wheat region. Consistent with the previous studies, winter wheat yield in China exhibited negative responses to higher daytime temperature $T_{\max }$. As a result of these opposing temperature effects on yield, the net economic impact of weather variations on China's winter wheat sector is uncertain and is sensitive to specifications and data. Average winter wheat yield is projected to decline by $5.3 \%-7.0 \%$ by 2050 under the global climate model HadGEM2-ES and by $2.0 \%-3.4 \%$ under the NorESM1-M model.
\end{abstract}

\section{Introduction}

Global observed temperature data over the past half century indicate that the daily minimum temperature $T_{\min }$ has been rising faster than the daily maximum temperature $T_{\max }$ in many parts of the world (Easterling et al. 1997). The asymmetric increases in $T_{\min }$ and $T_{\max }$ are expected to affect crop yields, because plant physiological processes such as photosynthesis and transpiration occur during daytime and are sensitive to changes in $T_{\max }$, whereas other physiological processes such as respiration occur throughout the day and are thus sensitive to changes in both $T_{\min }$ and $T_{\max }$ (Peng et al. 2013; Lobell and Ortiz-Monasterio 2007). Several studies focusing on rice production in Asia found that changes in $T_{\min }$ and $T_{\max }$ had large and significant impacts on rice yield (Welch et al. 2010; Peng et al. 2004; Chen et al. 2016a). Weather plays an important role in influencing wheat yield volatility (Albers et al. 2017) and agronomic studies have long suggested that wheat yield exhibits different responses to changes in $T_{\min }$ and

\footnotetext{
Corresponding author: Xiaoguang Chen, cxg@swufe.edu.cn
}

$T_{\max }$ (Porter and Gawith 1999; Lobell and OrtizMonasterio 2007). However, existing studies analyzing the temperature effects on wheat yield have primarily focused on examining the effects of rising daily average temperature $T_{\text {ave }}$ on yield [for a comprehensive review, see Liu et al. (2016)]. Hence, the impacts of higher $T_{\min }$ and $T_{\max }$ on wheat yield have not been well understood (Prasad et al. 2008).

The purpose of this paper is to assess the effects of changes in $T_{\min }$ and $T_{\max }$ on winter wheat yield in China. Using estimated coefficients of weather variables, we then estimate the net economic impact that warming has imposed on China's winter wheat sector and project the future impacts of warming on average winter wheat yield in China. As the world's largest wheat-producing country, China produced around 126.3 million metric tons of wheat, which accounted for about $17 \%$ of the global wheat production in 2011 (http://faostat3.fao.org/ browse/Q/QC/E). Wheat is also China's second mostprevalent field crop; the first position is held by rice. China grows both spring wheat and winter wheat. We focus on winter wheat because it is widely produced across China's agricultural heartland (Fig. 1) and accounts for about $90 \%$ of the country's total wheat 


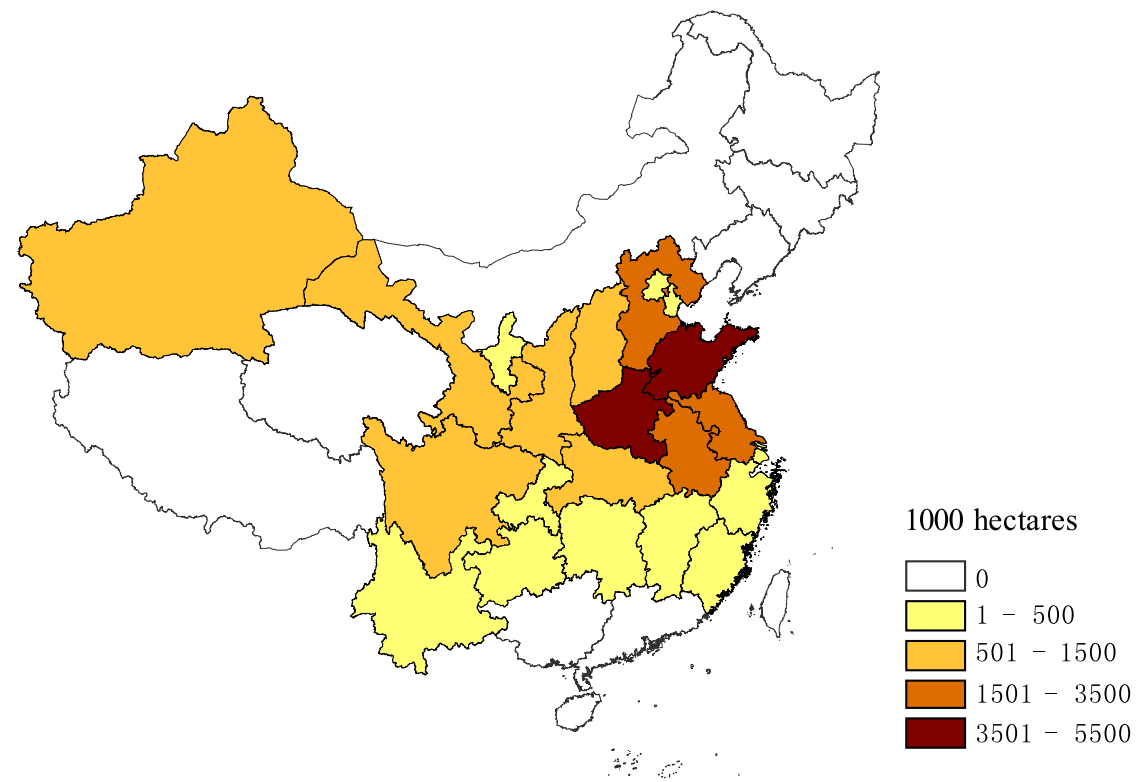

FIG. 1. Spatial distribution of winter wheat production in China in 2011 (in thousands of hectares).

production. Therefore, understanding how winter wheat yield has responded to rising $T_{\min }$ and $T_{\max }$ and estimating the extent to which warming has affected China's winter wheat sector provide useful information for the development of China's wheat-breeding programs and efficient adaptation strategies to cope with future warming.

Many studies have investigated the effects of temperature on wheat yield using the agronomic approach and the regression approach, at the global scale (Gourdji et al. 2013; Lobell and Field 2007) and at a regional scale (Nicholls 1997; Tack et al. 2015; Lobell et al. 2005). The agronomic approach mainly uses crop simulation models to simulate plant growth and predict how wheat yield will change under different warming scenarios. As compared to the agronomic approach, the regression approach does not consider the main physiological processes inherent to crop growth and thus cannot explain processes by which temperature affects crop yield (Liu et al. 2016). However, the regression approach may incorporate the effects of farmers' climate adaptation actions and extreme climate events, such as drought or flood, on yield.

Only a few studies have used the regression approach to assess the responses of wheat yield to the simultaneous variations in $T_{\min }$ and $T_{\max }$ [see a detailed reivew in Liu et al. (2016)]. From the data collected in a field trial at Kansas State University, Prasad et al. (2008) found a negative correlation between spring wheat yield and $T_{\min }$. Using historically observed yield and weather data, Lobell et al. (2005) and Lobell and Ortiz-Monasterio (2007) also showed that higher $T_{\min }$ has negatively affected spring wheat yield in Mexico. A few studies discovered that wheat yield exhibited negative responses to higher $T_{\max }$ (Gibson and Paulsen 1999; Tashiro and Wardlaw 1989; Lobell and OrtizMonasterio 2007; Zampieri et al. 2017).

Several studies have used the regression approach to assess the effects of variations in temperature on wheat yield in China (Xiong et al. 2014; You et al. 2009; Tao et al. 2008; Zhang et al. 2016; Li et al. 2010). However, these studies all have apparent methodological issues. As noted above, although wheat yield was found to exhibit different responses to changes in $T_{\min }$ and $T_{\max }$, most of these studies have used $T_{\text {ave }}$ as the temperature variable to examine variations in wheat yield (Xiong et al. 2014; Li et al. 2010; Zhang et al. 2016; You et al. 2009). Based on a provincial-scale dataset from 1979 to 2002, Tao et al. (2008) analyzed the effects of $T_{\min }$, $T_{\max }$ and precipitation on wheat yield. They showed that wheat yield was negatively correlated with $T_{\max }$ and that the effects of higher $T_{\min }$ on wheat yield varied across regions. However, the regression approach used by Tao et al. (2008) was problematic. When examining the effect of $T_{\min }$ (or $T_{\max }$ ) on wheat yield, they regressed wheat yield only on $T_{\min }$ (or $T_{\max }$ ), without controlling for other relevant weather variables. Weather variables, such as $T_{\min }, T_{\max }$, precipitation, and solar radiation, are highly correlated. If other weather 
variables were not considered when estimating the relationship between $T_{\min }$ (or $T_{\max }$ ) and yield, it would cause biased parameter estimates of the $T_{\min }$ (or $T_{\max }$ ) variable. Because of the methodological issues mentioned above, empirical findings reported in these earlier studies estimating the effects of temperature on wheat yield in China are questionable.

A few experimental studies have used the regression approach and the data collected from field trials to examine the impacts of higher $T_{\min }$ and $T_{\max }$ on wheat yield in China (Tao et al. 2017; Zheng et al. 2017). Researchers conducting these experimental studies typically apply agronomically optimal levels of inputs and do not consider the effects of nonclimate factors, such as prices of fertilizer, labor, and chemicals, on yield. As a result, their findings cannot represent outcomes that may occur in real agricultural settings (Welch et al. 2010).

In this paper, we develop a regression model to assess the responses of winter wheat yield to changes in $T_{\min }$ and $T_{\max }$. The regression model we developed incorporates a comprehensive set of weather variables, including $T_{\min }, T_{\max }$, precipitation, and solar radiation, enabling us to examine whether there exist differential effects of $T_{\min }$ and $T_{\max }$ on yield. Following Tack et al. (2015), we divide the growing cycle of winter wheat into three seasons: fall (from planting in late September or early October to November), winter (from December to February), and spring (from March to grain maturity). We construct these weather variables for each of the three seasons. We also incorporate input use in one model specification to investigate how winter wheat yield has responded to changes in input use. Moreover, we include various fixed effects to account for the unobserved factors that may have affected winter wheat yield. Specifically, we include region fixed effects to account for the time-invariant unobserved factors that are specific to a given region, such as geographical locations. We have also incorporated year fixed effects to account for the unobserved factors that had the same yield effects for all regions in a given year, such as global carbon dioxide $\left(\mathrm{CO}_{2}\right)$ concentrations. The regression analysis is conducted by using a provincialscale dataset of observed winter wheat yield, combined with daily weather outcomes during the period 1979-2011.

This paper makes two major contributions to the related literature. First, we add to the sparse literature examining the effects of rising $T_{\min }$ and $T_{\max }$ on winter wheat yield by employing a detailed provincial-scale dataset for the world's largest wheat-producing country. Several studies have used county-level yield data to analyze the effects of weather on corn, soybean, and rice yields in China (Chen et al. 2016a,b; Chen and Chen 2018). While the county-level yield data are typically available for 10-15 years, the province-level data used in this analysis cover observed winter wheat yield for more than 30 years, which allows us to examine the longer-term impacts of rising $T_{\min }$ and $T_{\max }$ on winter wheat yield. Second, contrasting with the previous studies assessing the effects of variations in temperature on winter wheat yield in China, we recognize that wheat yield is sensitive to changes in $T_{\min }$ and $T_{\max }$ and that this sensitivity differs across phenological growth stages of wheat. We also construct weather variables accordingly, which enables us to detect any differential effects of temperature on yield across the three growing seasons of wheat.

The rest of the paper is organized as follows. Section 2 introduces the regression model. Section 3 describes data sources. Section 4 presents the main results. Section 5 projects future impacts of warming on winter wheat yield. Section 6 discusses our findings and limitations. Section 7 concludes.

\section{Regression model}

We assess the weather effects on winter wheat yield using the following regression model:

$$
Y_{i, t}=W_{i, t} \alpha+E_{i, t} \beta+\theta_{i}+\lambda_{t}+\varepsilon_{i, t},
$$

where $i$ indexes province and $t$ indexes year. Thus, $Y_{i, t}$ represents the average winter wheat yield in province $i$ and year $t$. The $W_{i, t}$ is a vector of weather variables, including the means of $T_{\min }$ and $T_{\max }$ and the sums of radiation and precipitation for each of the three growing seasons of winter wheat. Thus, we have a total of 12 weather variables. The term $E_{i, t}$ is a vector of economic variables denoting input use per hectare for winter wheat production in province $i$ and year $t$. Province fixed effects $\theta_{i}$ are incorporated to account for the unobserved variables that were time invariant and unique to province $i$ during the sample period, such as soil quality. Year fixed effects $\lambda_{t}$ are also included to account for the unobserved variables that had the same effects on yield in all provinces in a given year, such as changes in global $\mathrm{CO}_{2}$ concentrations. The error terms $\varepsilon_{i, t}$ denote other unobserved factors that are not included in Eq. (1). The term $\alpha$ is a vector of the parameters of interest and measures the marginal effects on winter wheat yield of each unit change in weather variables, holding other variables constant.

We allow $\varepsilon_{i, t}$ to be spatially correlated, due to the concern that some of the variables, such as production practices used by neighboring provinces and possible pest problems experienced in neighboring provinces in a particular growing season, may be spatially correlated; 
however, these variables are omitted as explanatory variables in Eq. (1). The error terms may also be correlated over time. To account for this, we cluster standard errors in two dimensions: within provinces and within years (Cameron et al. 2011). The former accounts for autocorrelation within each province, while the latter accounts for spatial correlation across contemporary provinces. We control for the heteroskedasticity of the error terms.

\section{Data sources}

Data used for this analysis are compiled from several sources. We obtain province average yields of winter wheat from the National Bureau of Statistics of China for the period 1979-2011 (http://data.stats.gov.cn/ easyquery.htm?cn=E0103, last accessed on 13 May 2019). Figure 1 shows that, of the 34 provinces and provinceequivalent municipal cities in China, about 22 provinces produce winter wheat. Growing cycles of winter wheat in these 22 provinces are taken from the Ministry of Agriculture of the People's Republic of China, which reports rough estimates of planting and harvest dates of major crops produced in China. As summarized in Table 1, winter wheat is typically planted in late fall or early winter and harvested before summer. We collect per-unit inputs used for winter wheat production during the sample period, including machinery [cost in renminbi (RMB) ha ${ }^{-1}$ ], pesticide $\left(\mathrm{RMB} \mathrm{ha}^{-1}\right)$, fertilizer $\left(\mathrm{RMB} \mathrm{ha}^{-1}\right)$, irrigation $\left(\mathrm{RMB} \mathrm{ha}^{-1}\right)$, and labor (day ha ${ }^{-1}$ ) from the National Agricultural Product Cost-Benefit Data Compilation, 1980-2012 (http:// data.cnki.net/yearbook/Single/N2013010019, last accessed on 13 May 2019). Our sample is a balanced panel with 726 observations covering 22 provinces.

We obtain daily records of $T_{\min }, T_{\max }$, precipitation, and radiation for about 800 weather stations located in mainland China from the China Meteorological Data Sharing Service System. The daily weather data are merged with the yield data using the latitudes and longitudes of weather stations and province boundaries. We construct area-weighted province-level weather variables using weather information from weather stations in a province, with county-level availability of cropland in that province in 2010 as the weights. County-level cropland availability in 2010 is also compiled from the National Bureau of Statistics of China.

Table 2 reports summary statistics for winter wheat yield, weather variables, and input use. We find that winter wheat yield varied substantially across provinces and years, ranging between 537 and $6277 \mathrm{~kg} \mathrm{ha}^{-1}$. The average yield of winter wheat was $3024 \mathrm{~kg} \mathrm{ha}^{-1}$
TABLE 1. Growing cycles of winter wheat in China (source: http:// www.docin.com/p-624347927.html).

\begin{tabular}{ll}
\hline \multicolumn{1}{c}{ Growing cycle } & \multicolumn{1}{c}{ Province(s) } \\
\hline September-June & $\begin{array}{c}\text { Beijing, Gansu, Hebei, Shaanxi, Ningxia, } \\
\text { and Tianjin } \\
\text { Xinjiang } \\
\text { September-August } \\
\text { October-May }\end{array}$ \\
$\begin{array}{l}\text { Anhui, Chongqing, Guizhou, Hubei, } \\
\text { Hunan, Jiangsu, Jiangxi, Shanghai, } \\
\text { Sichuan, Yunnan, and Zhejiang }\end{array}$ \\
October-June & Henan, Shandong, and Shanxi \\
November-April & Fujian \\
\hline
\end{tabular}

during the sample period. Weather variables, including temperature, precipitation, and solar radiation, also exhibited considerable variability during the sample period.

Table 3 shows that the correlations of the weather variables are large and statistically significant during the three growing seasons of winter wheat. These test results suggest that, to isolate the direct impact of temperature on winter wheat yield, other correlated weather variables should be included as explanatory variables in Eq. (1). For instance, if the relationship between $T_{\min }$ and yield were estimated without including $T_{\max }$, precipitation, and solar radiation as explanatory variables, it would be difficult to tell whether the estimated coefficients of the $T_{\min }$ variables capture the effect on yield solely due to the changes in $T_{\min }$, as opposed to measuring the overall weather effects on yield, with $T_{\min }$ serving as a proxy rather than an explanatory variable.

The winter wheat growing region in China can be divided into five subregions, including the northern winter wheat region, the Huang-Huai River winter wheat region, the middle and lower Yangtze River winter wheat region, the southwestern winter wheat region, and the southern China winter wheat region (Huang et al. 2015). Scatterplots between $T_{\min }$ and winter wheat yield in Fig. 2 depict that higher $T_{\text {min }}$ is likely to positively affect winter wheat yield across the five subregions, with one exception in the middle and lower Yangtze River winter wheat region, where the statistical correlation between $T_{\min }$ in the spring and winter wheat yield is negative.

\section{Regression results}

\section{a. Baseline estimates}

In the empirical analysis, we consider three model specifications. In model 1 , we incorporate only $T_{\min }, T_{\max }$, and precipitation as weather controls. In model 2 , we add the radiation variables. In model 3, we incorporate the five economic variables to assess the yield responses to 
TABLE 2. Summary statistics, showing means for $T_{\min }$ and $T_{\max }$ and sums for radiation and precipitation. The sample period is from 1979 to 2011 . The number of observations is 726 .

\begin{tabular}{|c|c|c|c|c|}
\hline Variable & Mean & Std dev & Max & Min \\
\hline Yield $\left(\mathrm{kg} \mathrm{ha}^{-1}\right)$ & 3023.98 & 1271.74 & 6276.69 & 536.67 \\
\hline$T_{\min }:$ fall $\left({ }^{\circ} \mathrm{C}\right)$ & 9.02 & 3.67 & 18.07 & 0.41 \\
\hline$T_{\min }:$ winter $\left({ }^{\circ} \mathrm{C}\right)$ & -1.17 & 5.91 & 11.85 & -16.15 \\
\hline$T_{\min }$ spring $\left({ }^{\circ} \mathrm{C}\right)$ & 10.96 & 2.23 & 16.20 & 4.24 \\
\hline$T_{\max }:$ fall $\left({ }^{\circ} \mathrm{C}\right)$ & 18.41 & 2.21 & 23.84 & 11.80 \\
\hline$T_{\max }:$ winter $\left({ }^{\circ} \mathrm{C}\right)$ & 7.73 & 4.49 & 19.44 & -4.71 \\
\hline$T_{\max }:$ spring $\left({ }^{\circ} \mathrm{C}\right)$ & 21.22 & 2.03 & 25.79 & 15.92 \\
\hline Precipitation: fall (mm) & 99.2 & 58.69 & 366.03 & 0.97 \\
\hline Precip & 70.9 & 75.86 & 471. & 0.0 \\
\hline Precip & 233.63 & 135.50 & 903.49 & 42.21 \\
\hline Radia & 368.40 & 172.05 & 744.83 & 69.39 \\
\hline Radiation: $\mathrm{w}$ & 404.18 & 142.22 & 681.74 & 77.89 \\
\hline Radiation: spring (h) & 666.27 & 331.26 & 1705.15 & 113.70 \\
\hline Labor $\left(\right.$ day ha $\left.{ }^{-1}\right)$ & 194.26 & 105.22 & 592.50 & 42.00 \\
\hline Machinery $\left(\mathrm{RMB} \mathrm{ha}^{-1}\right)$ & 345.81 & 429.70 & 1896.90 & 0.30 \\
\hline Pesticide $\left(\mathrm{RMB} \mathrm{ha}^{-1}\right)$ & 68.75 & 69.04 & 385.05 & 0.15 \\
\hline Fertilizer $\left(\mathrm{RMB} \mathrm{ha}^{-1}\right)$ & 667.89 & 535.42 & 2456.85 & 16.15 \\
\hline Irrigation $\left(\mathrm{RMB} \mathrm{ha}^{-1}\right)$ & 150.02 & 217.31 & 1058.70 & 0.45 \\
\hline
\end{tabular}

changes in input use. Table 4 reports estimated effects of weather on winter wheat yield. We find that winter wheat yield responded positively to higher $T_{\min }$ in the fall and spring. Each $1^{\circ} \mathrm{C}$ increase in $T_{\min }$ in the fall was associated with a yield increase of 160 $185 \mathrm{~kg} \mathrm{ha}^{-1}$, depending on specifications. Relative to the yield increase in the fall, the positive effect on yield stemming from higher $T_{\min }$ in the spring is significantly larger. Holding all else the same, each $1^{\circ} \mathrm{C}$ increase in $T_{\min }$ in the spring is expected to increase winter wheat yield by $219-461 \mathrm{~kg} \mathrm{ha}^{-1}$, which is $19 \%-$ $188 \%$ larger than the corresponding coefficient estimates in the fall. The estimated impact on yield of higher $T_{\min }$ in the winter is also positive, but is not statistically significant.

We find that winter wheat yield responded negatively to elevated $T_{\max }$, with the negative yield impacts varying across specifications and wheat growing seasons. In model 1 , with temperature and precipitation as weather variables, higher $T_{\max }$ had large and negative impacts on winter wheat yield during the three growing seasons. The negative impacts of higher $T_{\max }$ on yield are statistically significant at the $5 \%$ level in the fall and spring and at the $10 \%$ level in the winter. For each $1^{\circ} \mathrm{C}$ increase in $T_{\max }$, the estimated $T_{\max }$ effect ranges from $-108 \mathrm{~kg} \mathrm{ha}^{-1}$ in the fall to $-178 \mathrm{~kg} \mathrm{ha}^{-1}$ in the spring. In models 2 and 3 , with the inclusion of the three radiation variables, the negative effects of $T_{\max }$ on yield are only statistically significant in the fall and spring. Specifically, each $1^{\circ} \mathrm{C}$ increase in $T_{\max }$ in the fall and spring reduces winter wheat yield by $109-145$ and $326-451 \mathrm{~kg} \mathrm{ha}^{-1}$,
TABLE 3. Correlations of weather variables during the three growing seasons of winter wheat (three asterisks indicates $p<0.01$; two asterisks shows $p<0.05$ ).

\begin{tabular}{|c|c|c|c|c|}
\hline Growing season & Variable & $T_{\min }$ & $T_{\max }$ & Radiation \\
\hline \multirow[t]{3}{*}{ Fall } & $T_{\max }$ & $0.35^{* * * *}$ & - & - \\
\hline & Radiation & $-0.30^{* * * *}$ & $0.59^{* * *}$ & - \\
\hline & Precipitation & $0.19^{\text {**** }}$ & $-0.45^{\text {**** }}$ & $-0.51^{\text {***** }}$ \\
\hline \multirow[t]{3}{*}{ Winter } & $T_{\max }$ & $0.58^{* * * *}$ & - & - \\
\hline & Radiation & $-0.07^{* *}$ & $0.57^{\text {**** }}$ & - \\
\hline & Precipitation & 0.05 & $-0.17^{* * * *}$ & $-0.37^{* * * *}$ \\
\hline \multirow[t]{3}{*}{ Spring } & $T_{\max }$ & $0.61^{* * * *}$ & - & - \\
\hline & Radiation & 0.06 & $0.65^{\text {**** }}$ & - \\
\hline & Precipitation & $-0.19^{* * * k}$ & $-0.44^{* * * *}$ & $-0.33^{* * * *}$ \\
\hline
\end{tabular}

respectively. Across the three model specifications that we considered, we find that precipitation had negligible impacts on winter wheat yield.

The addition of the radiation variables in model 2 affects both magnitudes and significance levels of the coefficient estimates of temperature variables. Specifically, the inclusion of radiation decreases the parameter estimate of the $T_{\min }$ variable in the fall by $13 \%$, while increasing the parameter estimate of $T_{\min }$ in the spring by $110 \%$. Higher $T_{\max }$ is found to have significant negative effects on winter wheat yield across the three growing seasons in model 1 . The negative responses are only significant in the fall and spring in model 2 . The inclusion of the radiation variables in model 2 also increases the absolute value of the parameter estimate of $T_{\max }$ in the spring by $154 \%$ relative to the corresponding parameter estimate in model 1 . These findings are expected, given the large correlations between temperature and radiation during the wheat growing period (Table 3). Increased radiation in the spring had a significant and positive effect on winter wheat yield, while the radiation impacts on winter wheat yield are insignificant in the fall and winter.

Estimated weather effects on winter wheat yield remain broadly consistent when the five economic variables are added in model 3. Coefficient estimates of the labor, fertilizer, and irrigation variables are positive and statistically significant at the $10 \%$ level, indicating that increased use of labor, fertilizer, and irrigation may have effectively boosted winter wheat yield. The coefficient estimates of the machinery and pesticide variables are insignificant.

\section{b. Robustness checks}

We consider three scenarios to examine the robustness of estimated temperature effects on winter wheat yield. In scenario 1 , we include year as a linear time 

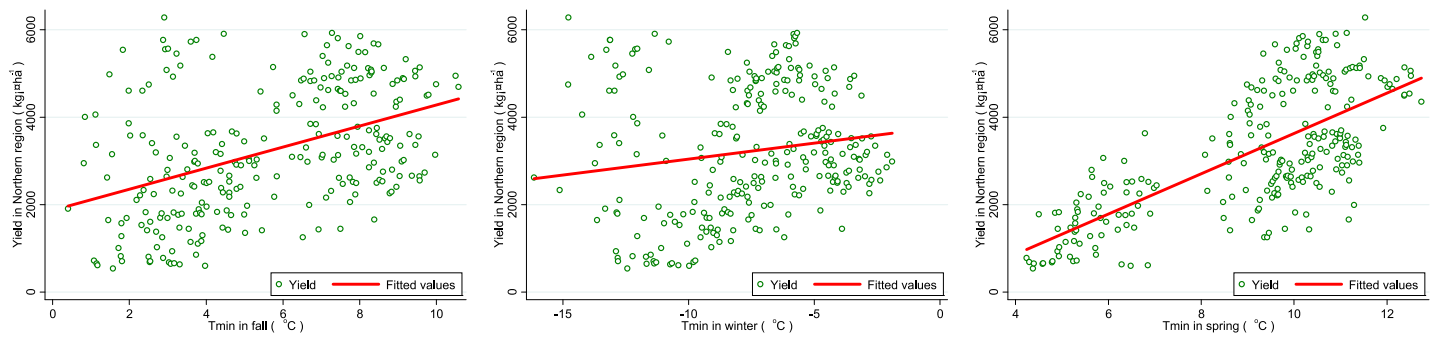

(a) Northern winter wheat region
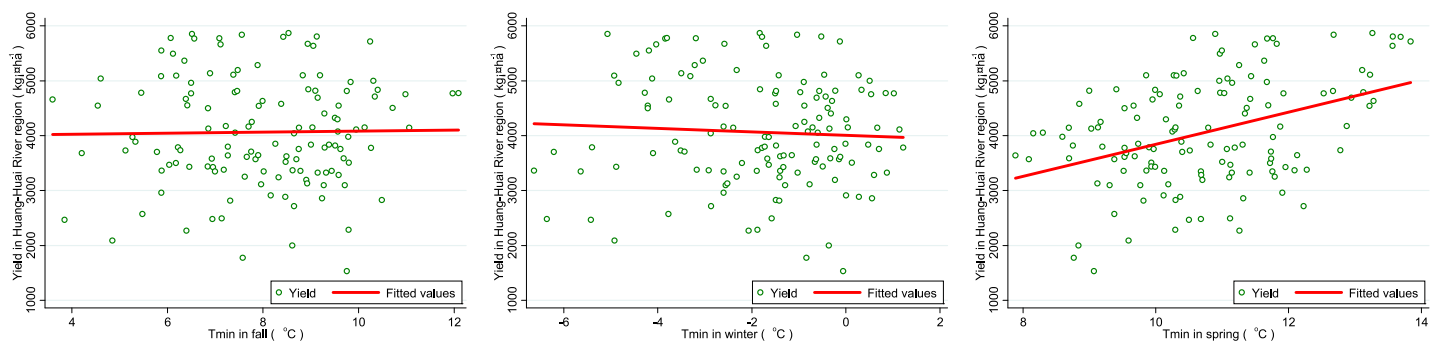

(b) Huang-Huai River winter wheat region
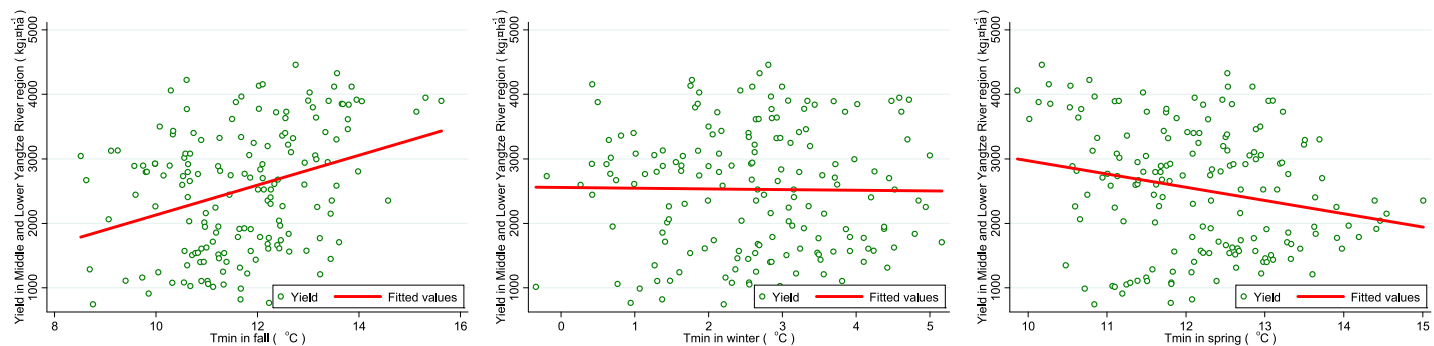

(c) Middle and Lower Yangtze River winter wheat region
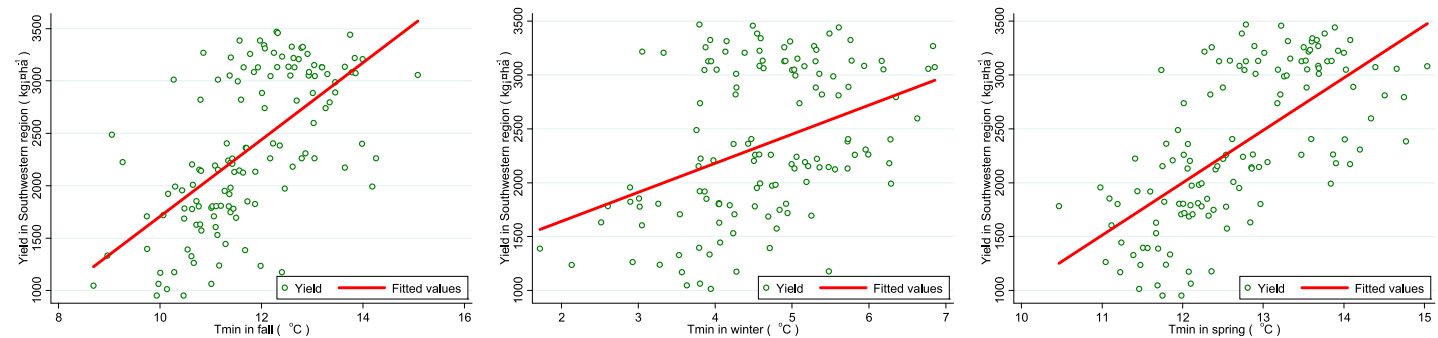

(d) Southwestern winter wheat region
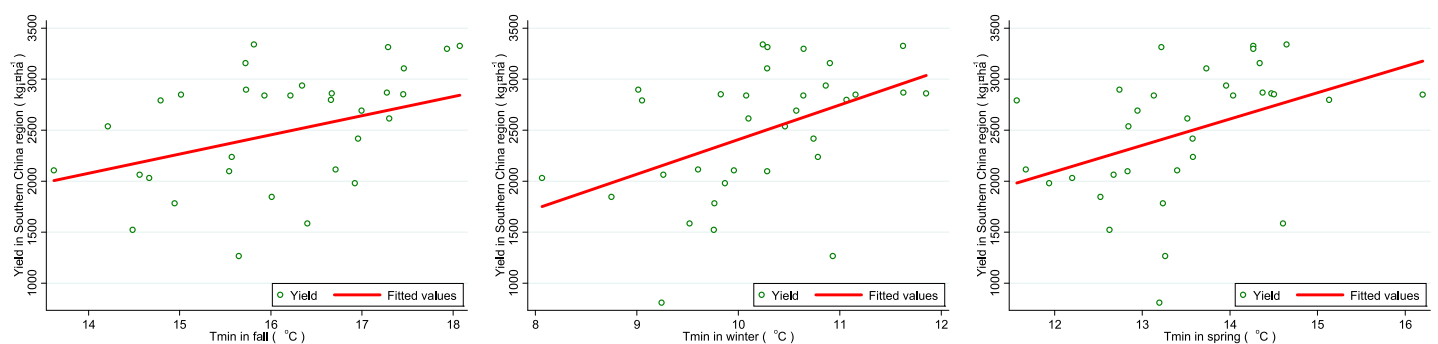

(e) Southern China winter wheat region

FIG. 2. Scatterplots of winter wheat yield vs $T_{\min }$ across winter wheat growing seasons [(left) fall, (center) winter, and (right) spring] and regions (rows). Note that the yield axis scale varies by region and the temperature axis scale varies by panel. 
TABLE 4. Main regression results. Coefficient estimates of weather variables are obtained by fitting Eq. (1). Parameter estimates are interpreted as the marginal effects of per-unit change in the temperature $\left({ }^{\circ} \mathrm{C}\right)$, precipitation $(\mathrm{mm})$, and radiation $(\mathrm{h})$ variables on yield. Standard errors, adjusted for heteroskedasticity, autocorrelation, and spatial correlation of the error terms, are shown in parentheses; $R^{2}$ is the correlation coefficient squared; three asterisks indicates $p<0.01$, two asterisks shows $p<0.05$, and one asterisk gives $p<0.1$.

\begin{tabular}{|c|c|c|c|}
\hline Variables & Model 1 & Model 2 & Model 3 \\
\hline$T_{\min }:$ fall & $184.68^{* * *}(69.62)$ & $159.81^{* * *}(60.70)$ & $176.31^{\text {**** }}(54.85)$ \\
\hline$T_{\min }:$ winter & 206.27 (125.69) & $140.83(117.72)$ & $126.88(84.39)$ \\
\hline$T_{\min }:$ spring & $219.08^{*}(126.60)$ & $461.00^{* * * *}(155.57)$ & $265.92^{* * *}(96.75)$ \\
\hline$T_{\max }:$ fall & $-108.47^{* * *}(46.57)$ & $-108.88^{* * *}(44.10)$ & $-144.95^{* * * *}(38.06)$ \\
\hline$T_{\max }:$ winter & $-120.735^{*}(63.33)$ & $-37.66(54.71)$ & $-27.79(52.60)$ \\
\hline$T_{\max }:$ spring & $-177.89^{* *}(79.64)$ & $-451.02^{* * * *}(127.62)$ & $-325.53^{* * *}(76.49)$ \\
\hline Precipitation: fall & $0.24(0.72)$ & $0.22(0.79)$ & $0.42(0.66)$ \\
\hline Precipitation: winter & $-3.30^{* * * *}(1.09)$ & $-3.34^{* * * *}(1.15)$ & $-3.07^{* * * *}(0.88)$ \\
\hline Precipitation: spring & $-1.00^{* *}(0.38)$ & $-0.98^{* * *}(0.46)$ & $-0.93^{* *}(0.41)$ \\
\hline Radiation: fall & & $-0.30(1.09)$ & $0.76(1.03)$ \\
\hline Radiation: winter & & $-1.16(0.95)$ & $-0.66(0.96)$ \\
\hline Radiation: spring & & $3.45^{\text {**** }}(0.98)$ & $2.27^{* * * *}(0.55)$ \\
\hline Labor $\left(\right.$ day ha $\left.{ }^{-1}\right)$ & & & $1.45^{*}(0.81)$ \\
\hline Machinery $\left(\mathrm{RMB} \mathrm{ha}^{-1}\right)$ & & & $0.34(0.21)$ \\
\hline Pesticide $\left(\mathrm{RMB} \mathrm{ha}^{-1}\right)$ & & & $-0.33(1.08)$ \\
\hline Fertilizer $\left(\mathrm{RMB} \mathrm{ha}^{-1}\right)$ & & & $0.42^{*}(0.21)$ \\
\hline Irrigation $\left(\mathrm{RMB} \mathrm{ha}^{-1}\right)$ & & & $0.71^{*}(0.41)$ \\
\hline$R^{2}$ & 0.593 & 0.611 & 0.695 \\
\hline
\end{tabular}

trend in the regression to remove the effects on yield of possible confounding factors, such as agricultural management technology, improvement of seed quality, and perhaps others, rather than the year fixed effects specified in the baseline model. In scenario 2, we remove provinces with extremely low yields from the sample to ensure that our estimates of the temperature effects on yield are not subject to estimation bias due to potentially mis-specified outliers. In scenario 3, we follow the standard agronomic literature and redefine growing seasons of winter wheat ("Wheat growth and physiology," FAO corporate document repository; available online at http://www.fao.org/docrep/006/Y4011E/y4011e06.htm). Specifically, we divide the growing cycle of winter wheat into three growth phases, including the vegetative growth phase from establishment to tillering, the reproductive growth phase from tillering to heading, and the maturation growth phase from heading to mature grain. Based on the three growth stages, we reconstruct weather variables and replicate the above regression analysis using the newly constructed weather variables. These sensitivity analyses are conducted using model 3.

Figure 3 displays the results of our robustness checks. To facilitate comparisons, Fig. 3 also presents our baseline estimates reported in the last column in Table 4. Our key findings of the positive $T_{\min }$ effects and the negative $T_{\max }$ effects on winter wheat yield remain broadly consistent across the three scenarios. We notice that the positive $T_{\min }$ effects are statistically significant in the fall and spring in the baseline scenario. In scenario 1 , the positive $T_{\min }$ effects on yield are statistically significant in the winter and spring; the effect of higher $T_{\min }$ in the fall on winter wheat yield is still positive but insignificant. In scenario 2, the estimated $T_{\min }$ impacts are similar to our baseline estimates. In scenario 3 , the positive $T_{\min }$ effects on yield are statistically significant only during the vegetative stage.

Across the various scenarios that we considered, we find that higher $T_{\max }$ in the fall had significant negative impacts on winter wheat yield, with an exception in scenario 1 , where the estimated $T_{\max }$ impact on yield is negative but insignificant. Higher $T_{\max }$ also exerted negative impacts on winter wheat yield near the time of grain maturity in the spring (or during the maturation stage). Estimated precipitation and radiation impacts on winter wheat yield in scenarios 1-3 are broadly consistent with our baseline estimates.

\section{c. Heterogeneous temperature effects on winter wheat yield}

The estimated temperature impacts on winter wheat yield may differ across regions due to the differences in exposure to high temperatures, wheat resilience to temperature variations, and/or crop management practices. To examine the heterogeneity in temperature effects across regions, we use model 3 with the inclusion of he full set of weather and input-use 


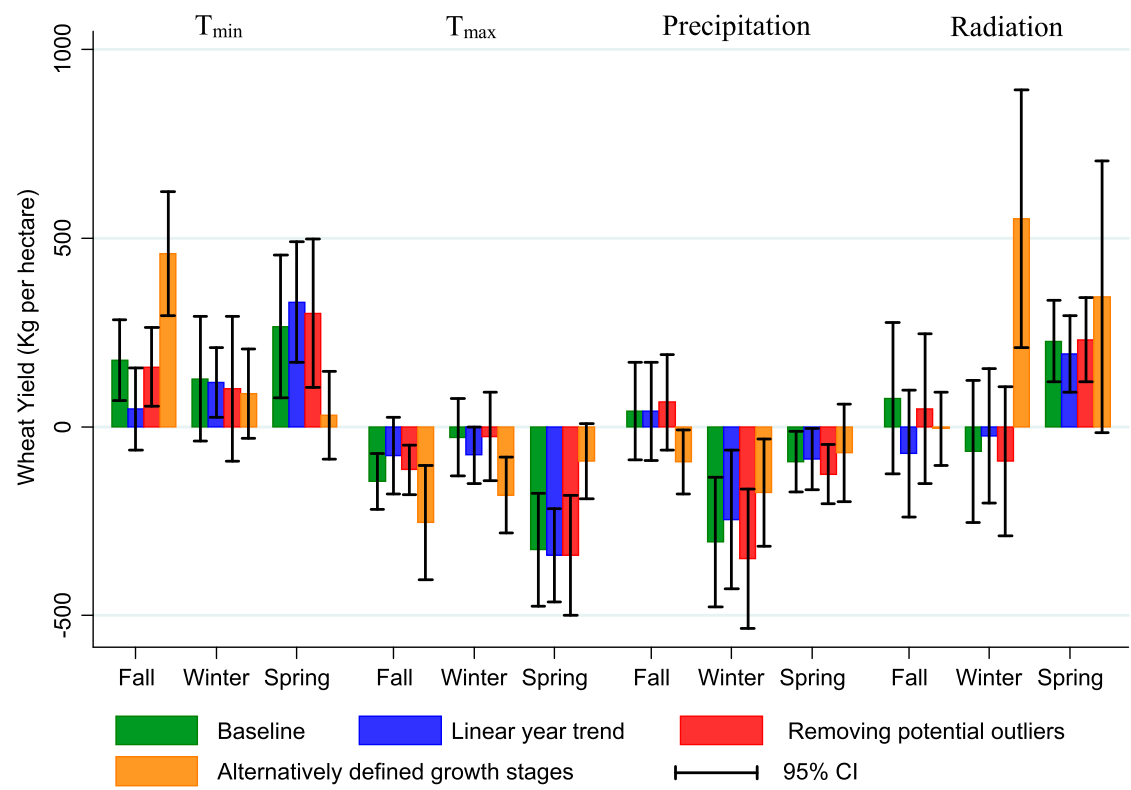

FIG. 3. Impacts of weather variations on winter wheat yield across three growing seasons (fall, winter, and spring) under different scenarios. Each cluster shows the impacts of a given variable on yield, varied by winter wheat growing season. Parameter estimates are interpreted as the marginal effects of per-unit change in the temperature $\left({ }^{\circ} \mathrm{C}\right)$, precipitation $(10 \mathrm{~cm})$, and radiation $(100 \mathrm{~h})$ variables on yield $\left(\mathrm{kg} \mathrm{ha}^{-1}\right)$. Error bars show $95 \%$ confidence bands. In the scenario "alternatively defined growth stages" fall denotes the vegetative stage from germination/emergence to tillering, winter denotes the reproductive stage from tillering to heading, and spring denotes the maturation stage from heading to grain maturity.

variables to replicate the aforementioned regression analyses for each of the five winter wheat producing subregions.

Figure 4a displays the temperature effects on winter wheat yield by region. We find that winter wheat yield in the northern winter wheat region responded positively to higher $T_{\min }$ in the fall and spring. Specifically, each $1^{\circ} \mathrm{C}$ increase in $T_{\min }$ in the fall and spring was associated with a yield increase of 390 and $411 \mathrm{~kg} \mathrm{ha}^{-1}$, respectively, in the northern winter wheat region. These results are consistent with our baseline findings presented above based on the full sample. Higher $T_{\min }$ also had positive impacts on yield in other winter wheat growing regions, but these impacts are not statistically significant.

Elevated $T_{\max }$ had negative effects on winter wheat yield in the northern winter wheat region, with the negative $T_{\max }$ effects occurring in the fall and spring. Specifically, holding all else equal, each $1^{\circ} \mathrm{C}$ increase in $T_{\max }$ in the fall is expected to reduce winter wheat yield in the northern winter wheat region by $227 \mathrm{~kg} \mathrm{ha}^{-1}$, while winter wheat yield in this region declines $482 \mathrm{~kg} \mathrm{ha}^{-1}$ for each $1^{\circ} \mathrm{C}$ increase in $T_{\max }$ in the spring. The effects of higher $T_{\max }$ on winter wheat yield in other regions are either insignificant or exhibit low levels of statistical significance. For instance, higher $T_{\max }$ in the fall is estimated to reduce winter wheat yield in the Huang-Huai River winter wheat region by $338 \mathrm{~kg} \mathrm{ha}^{-1}$, while $1^{\circ} \mathrm{C}$ higher $T_{\max }$ in the winter led to a yield reduction of $278 \mathrm{~kg} \mathrm{ha}^{-1}$ in the middle and lower Yangtze River winter wheat region. The two estimates of the negative $T_{\max }$ effects on yield are statistically significant only at the $10 \%$ level. Consistent with our baseline findings based on the full sample, we find modest precipitation and radiation impacts on yield.

The estimated relationships between temperature and winter wheat yield may also differ under irrigated and rainfed conditions. To probe this, we split the full sample into two subsamples based on whether the production of winter wheat is irrigated or rainfed. Figure $4 \mathrm{~b}$ depicts that the yield responses of irrigated winter wheat to higher $T_{\text {min }}$ differ from those of rainfed winter wheat. Specifically, for each $1^{\circ} \mathrm{C}$ increase in $T_{\min }$ in the fall and spring, irrigated winter wheat yield increases 256 and $388 \mathrm{~kg} \mathrm{ha}^{-1}$, respectively, holding all else the same. In contrast, winter wheat yield under rainfed conditions did not exhibit strong responses to higher $T_{\min }$ across the three growing seasons of winter wheat. 


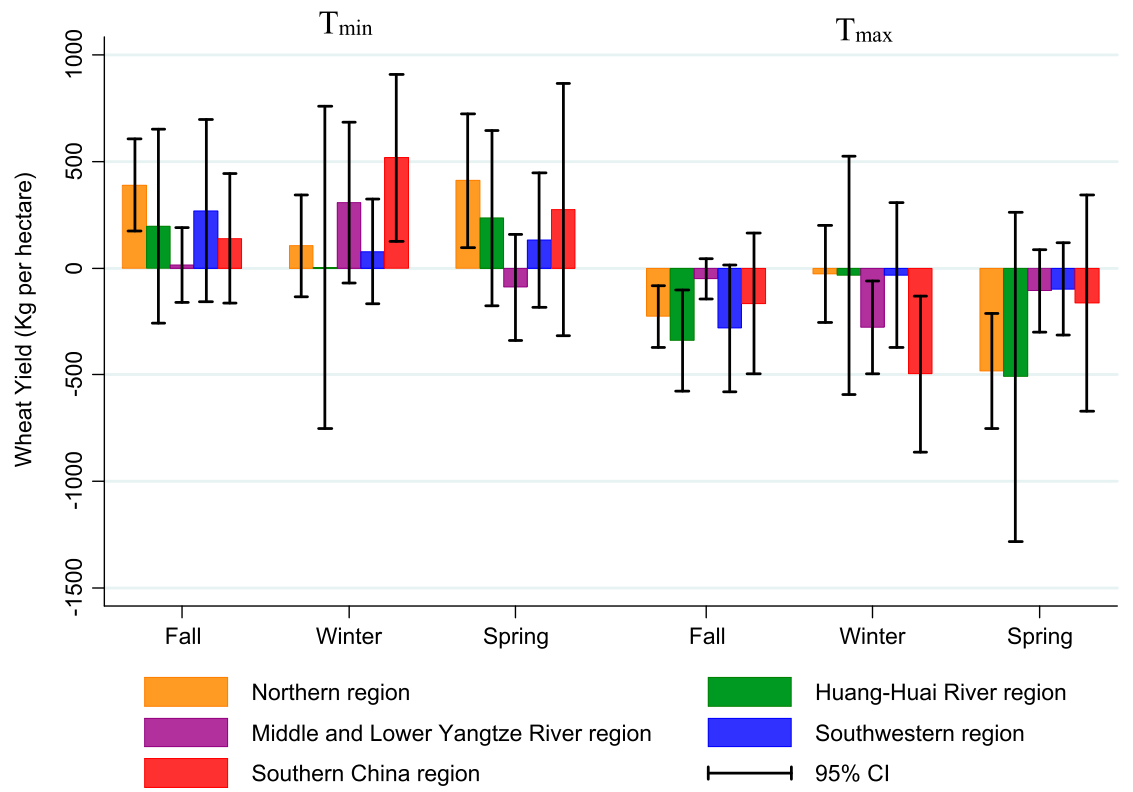

(a) Across major winter wheat producing regions

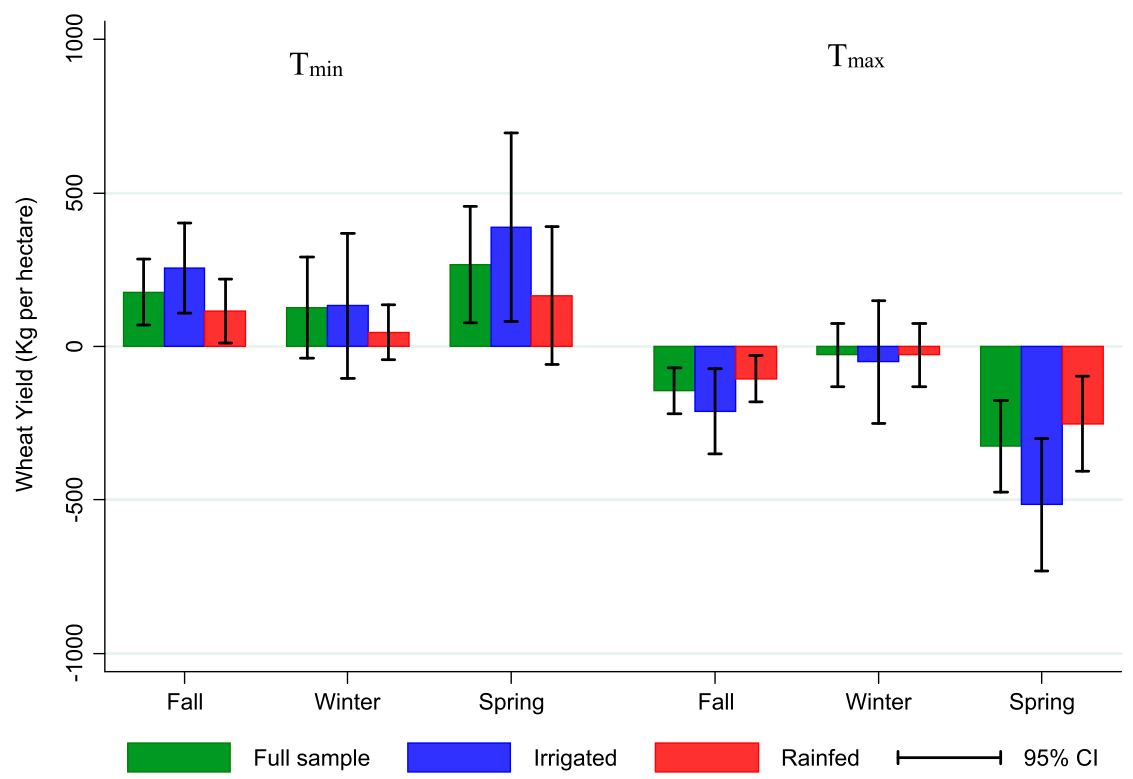

(b) Under irrigated and rainfed conditions

FIG. 4. Heterogeneous temperature effects on winter wheat yield. Each cluster shows the impacts of a given variable on yield, varied by region. Parameter estimates are interpreted as the marginal effects of per-unit change in the temperature $\left({ }^{\circ} \mathrm{C}\right)$, precipitation $(10 \mathrm{~cm})$, and radiation $(100 \mathrm{~h})$ variables on yield $\left(\mathrm{kg} \mathrm{ha}^{-1}\right)$. Error bars show $95 \%$ confidence bands.

Our parameter estimates of the $T_{\max }$ variables suggest that higher $T_{\max }$ in the fall and spring exerted significant negative impacts on winter wheat yield under both irrigated and rainfed conditions. Holding all else equal, irrigated winter wheat yield declines $212 \mathrm{~kg} \mathrm{ha}^{-1}$ for each $1^{\circ} \mathrm{C}$ increase in $T_{\max }$ in the fall and $516 \mathrm{~kg} \mathrm{ha}^{-1}$ for each $1^{\circ} \mathrm{C}$ increase in $T_{\max }$ in the spring. In comparison, the negative responses of winter wheat yield to higher $T_{\max }$ under rainfed conditions are smaller, about $50 \%$ of the magnitude of the negative responses of irrigated winter wheat yield to higher $T_{\max }$. 


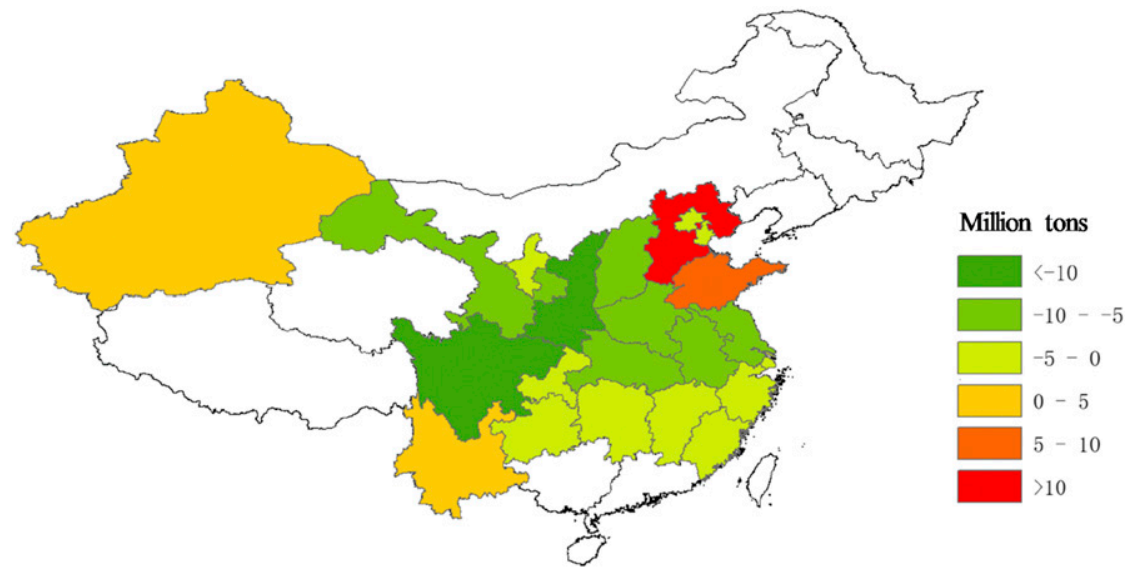

FIG. 5. Spatial changes in winter wheat production from weather variations over the 1980 2011 period. The green areas denote reductions in total winter wheat production during the 1980-2011 period resulting from weather variations, and the yellow areas denote increases in total winter wheat production over the same period. These estimates are based on the baseline parameter estimates of weather variables that are reported in the rightmost column of Table 4 .

\section{d. Assessing the economic impact of warming on China's winter wheat sector}

In this section, we use estimated coefficients of weather variables to quantify the net economic impact of warming on China's winter wheat sector during the sample period. Coefficient estimates of weather variables used in this analysis are based on model 3 and include our baseline estimates and the estimates obtained in the robustness check section (these parameter estimates are shown in Fig. 3). To proceed, we first use estimated coefficients of weather variables to compute the province-specific changes in winter wheat yields for 1980-2011 stemming from changing weather conditions relative to 1979 :

$$
\Delta Y_{i, t}=E\left(\hat{Y} \mid W_{i, 1979}, S_{i, t}\right)-E\left(\hat{Y} \mid W_{i, t}, S_{i, t}\right),
$$

where $E\left(\hat{Y} \mid W_{i, 1979}, S_{i, t}\right)$ denotes the expected winter wheat yields with the weather conditions in 1979 and economic variables in year $t$ and $E\left(\hat{Y} \mid W_{i, t}, S_{i, t}\right)$ represents the expected winter wheat yields when all explanatory variables are at their observed levels in year $t$. Therefore, $\Delta Y_{i, t}$ measures the province-specific changes in winter wheat yields due to weather variations.

We then multiply $\Delta Y_{i, t}$ by province-level winter wheat planted acres, summed across all provinces and years, to obtain an estimate of the change in total winter wheat production resulting from weather variations. Last, we multiply the change in total wheat production by the market price of wheat in 2011 (http://www.fao.org/giews/ food-prices/tool/public/\#/dataset/domestic) and subtract associated production costs to obtain a rough estimate of the net economic impact of changing weather conditions on China's winter wheat sector during the past three decades.

We find that the overall economic impact of weather variations on China's winter wheat sector is uncertain and sensitive to model specifications and the scenarios we considered. If we use the baseline estimates of the weather variables, we find that, of the 22 winter wheat-producing provinces in China, changing weather conditions over the past three decades caused net reductions in total winter wheat production in 18 provinces (the green areas in Fig. 5), while the winter wheat production in the rest of the regions (the yellow areas in Fig. 5) benefited from elevated temperatures. This is an expected outcome because $T_{\min }$ and $T_{\max }$ exerted opposing impacts on winter wheat yield and these impacts varied across regions. If we use the baseline estimates of the weather variables, the net economic impact of weather variation is negative and equal to $-\$ 38.7$ billion in 2011 prices. When using the coefficient estimates of the weather variables obtained from the scenarios in the robustness check section, the net economic impact ranges between $\$-110.0$ billion in scenario 3 and $\$ 39.1$ billion in scenario 1 .

\section{Future impacts of warming}

In this section, we use our baseline estimates of temperature variables based on model 3 to project the potential impacts of future warming on winter wheat yield. Projections of future climate variables are taken from WorldClim (http://www.worldclim.org/; last accessed 28 September 2018), which provides monthly climate 
TABLE 5. Predicted changes in temperature in the medium term. This table reports descriptive statistics of projected changes in temperature variables under the two pathways (RCP 2.6 and RCP 8.5) from the climate models HadGEM2-ES and NorESM1-M in the medium term (2050s). Projected changes are computed by using the projected $T_{\max }$ and $T_{\min }$ minus average $T_{\max }$ and $T_{\min }$ in our sample. The numbers in parentheses are standard deviations.

\begin{tabular}{|c|c|c|c|c|}
\hline & \multicolumn{2}{|c|}{$T_{\max }$} & \multicolumn{2}{|c|}{$T_{\min }$} \\
\hline & HadGEM2-ES & NorESM1-M & HadGEM2-ES & NorESM1-M \\
\hline \multicolumn{5}{|c|}{ RCP 2.6} \\
\hline Fall & $3.29(1.97)$ & 2.67 (1.77) & $2.77(2.21)$ & $2.52(2.18)$ \\
\hline Winter & $1.25(1.46)$ & $0.18(1.32)$ & $0.69(1.92)$ & $-0.35(1.80)$ \\
\hline Spring & $1.93(2.19)$ & $1.30(2.08)$ & $1.75(2.36)$ & $1.21(2.23)$ \\
\hline \multicolumn{5}{|c|}{ RCP 8.5} \\
\hline Fall & $4.30(2.00)$ & $3.32(1.85)$ & $3.78(2.19)$ & $3.22(1.96)$ \\
\hline Winter & $2.21(1.52)$ & $1.12(1.25)$ & $1.78(1.95)$ & $1.11(1.75)$ \\
\hline Spring & $2.90(2.08)$ & $2.20(2.11)$ & $2.73(2.25)$ & $2.24(2.24)$ \\
\hline
\end{tabular}

predictions in the medium term (average for 2041-60) and in the long term (average for 2061-80) based on climate projections from the most recent global climate models. The climate projection data provided by WorldClim were used in the IPCC Fifth Assessment report, and include monthly predictions on average minimum temperature, average maximum temperature, and total precipitation under four representative concentration pathways (RCPs). The four pathways are RCP2.6, RCP4.5, RCP6.0, and RCP8.5, which differ by assumed greenhouse gas (GHG) concentration trajectory. RCP2.6 assumes that global GHG emissions peak between 2010 and 2020 and decline quickly thereafter, whereas RCP8.5 assumes that GHG emissions continue to rise during this century. We select RCP2.6 and RCP8.5 for this analysis because the two pathways cover the entire range for the projected rates of future GHG emissions. Following Warszawski et al. (2014), we use climate data based on the global climate models HadGEM2-ES and NorESM1-M, which provide a good contrast on future global temperature changes. We utilize the data at the spatial resolution of 2.5 minutes of a degree of longitude and latitude, which enables us to obtain future climate variables for all Chinese winter wheat-producing provinces. We project the impact of future warming on winter wheat yield for the medium term only, because farmers may undertake adaptation measures in the long term to cope with future warming and the estimated effects of temperature on winter wheat yield based on the observations of the last three decades may not capture these adaptation actions.

Table 5 reports summary statistics of the projected changes in $T_{\min }$ and $T_{\max }$, which are computed by using temperature data based on the WordClim database minus sample means during the 1979-2011 period. HadGEM2-ES projects that, under RCP 2.6, average $T_{\max }$ during the three growing seasons of winter wheat would increase by $1.3^{\circ}-3.3^{\circ} \mathrm{C}$ in the medium term. The
$T_{\max }$ increases under $\mathrm{RCP} 8.5$ are about $1.0^{\circ} \mathrm{C}$ larger than the corresponding $T_{\max }$ increases under RCP 2.6. HadGEM2-ES projects that average $T_{\min }$ would also increase by $0.7^{\circ}-2.8^{\circ} \mathrm{C}$ under RCP 2.6 and by $1.8^{\circ}-$ $3.8^{\circ} \mathrm{C}$ under RCP 8.5 in the medium term. Relative to the climate model HadGEM2-ES, temperature increases predicted by NorESM1-M are slightly smaller.

We find that future warming reduces winter wheat yield and that the magnitudes of the yield reductions depend on climate models. If we use climate projections from the HadGEM2-ES model, in the medium term, average winter wheat yield in China is expected to decrease by $5.3 \%$ under RCP 2.6 and by $7.0 \%$ under RCP 8.5. If climate projections from the climate model NorESM1-M are utilized, average winter wheat yield in China is expected to decrease by $2.0 \%$ under RCP 2.6 and by $3.4 \%$ under RCP 8.5 by 2050 .

\section{Discussion}

Prior studies found negative correlations between $T_{\min }$ and spring wheat yield (Prasad et al. 2008; Lobell et al. 2005; Lobell and Ortiz-Monasterio 2007). In controlled environment facilities at Kansas State University, Prasad et al. (2008) discovered that increasing $T_{\min }$ from $14^{\circ}$ to $23^{\circ} \mathrm{C}$ during wheat's reproductive phase reduced spring wheat yield, mainly by decreasing photosynthesis, spikelet fertility, grains per spike, grain size, and grain filling duration. In Mexico, increased $T_{\min }$ was found to shorten the wheat growing season (Lobell and Ortiz-Monasterio 2007). Porter and Gawith (1999) reviewed literature describing the responses of wheat plants to temperature variations during the wheat growth and development stages. They found that higher $T_{\min }$ can negatively affect leaf initiation, tillering, and spikelet initiation, thus resulting in reduced wheat yield.

In this paper, we show that winter wheat yield in China exhibited positive responses to higher $T_{\min }$. 
One possible reason for the difference in findings between this paper and the prior studies is that $T_{\min }$ within our sample never reached the critical $T_{\min }$ threshold above which temperatures are detrimental for wheat yield. For instance, the optimal $T_{\min }$ for winter wheat growth during the maturation stage typically ranges between $18^{\circ}$ and $25^{\circ} \mathrm{C}$ (see Table 4 in Porter and Gawith 1999). In our sample, the average and maximum $T_{\min }$ in the spring were only $11.0^{\circ}$ and $16.2^{\circ} \mathrm{C}$, respectively (Table 2). When the ambient temperature is below the optimal $T_{\min }$ for winter wheat grain filling, a modest temperature increase can make the ambient temperature more suitable for the grain filling stage (Porter and Gawith 1999).

Our further analysis indicates that the positive $T_{\min }$ effects on yield are statistically significant only in China's northern winter wheat region, while the $T_{\min }$ effects are insignificant in other winter wheat growing regions. This finding is consistent with those reported in several recent experimental studies based on field trials, which all suggest that winter wheat yield in North China may increase with higher $T_{\min }$ (Fang et al. 2015; Chen et al. 2014; Fang et al. 2012). For instance, based on field experiments conducted in Hebei province in the North China Plain, Fang et al. $(2012,2015)$ showed that winter wheat yield benefited from higher $T_{\min }$, because higher nighttime temperatures caused an increase in plant height and panicle numbers, while reducing frost damage. Based on a 3-yr field experiment in North China, Chen et al. (2014) found that, by extending the duration of grain filling and promoting the filling rates of the superior and inferior grains, an increase of $1.1^{\circ} \mathrm{C}$ in average $T_{\min }$ led to a $12 \%$ increase in winter wheat yield.

The positive effect of higher $T_{\min }$ on winter wheat yield has also been observed in other major winter wheat producing regions in China. Tao et al. (2017) analyzed wheat trial data from 1981 to 2009, collected from 34 national agro-meteorological stations in China's HuangHuai River winter wheat region, and found a positive correlation between winter wheat yield and $T_{\min }$. The positive yield impact is mainly due to the fact that increased $T_{\min }$ reduced cold injury and frost damage to seedlings (Tao et al. 2017). Zheng et al. (2017) analyzed a dataset of winter wheat yield collected from four experimental sites in Hebei, Henan, and Jiangsu provinces between 1980 and 2015. Their results suggest that an increase of $1.0^{\circ} \mathrm{C}$ in $T_{\min }$ raised winter wheat yield by $10.1 \%$, which can be attributed to warminginduced enhancement of grain weight.

Our finding of the negative effects of $T_{\max }$ on winter wheat yield is consistent with the previous assessments (Gibson and Paulsen 1999; Tashiro and Wardlaw 1989;
Lobell and Ortiz-Monasterio 2007). Higher $T_{\max }$ can negatively affect winter wheat yield by reducing grain number and grain size (Gibson and Paulsen 1999; Lobell et al. 2012) and shortening the duration of wheat grain filling (Tashiro and Wardlaw 1989). As discussed above, our key findings of the positive $T_{\min }$ effects and the negative $T_{\max }$ effects on winter wheat yield remain broadly consistent across different model specifications.

Crop models have been widely used to evaluate the responses of crops to temperature variations over the growth cycle of the plant and to predict the impact of changed climatic conditions on crop yield. Compared with process-based crop models, statistical approaches are simpler in that they do not consider physiological processes inherent to crop growth (Liu et al. 2016). The effect of temperature on crop yield is highly complex, depending on growth stage, cultivar, soil quality, and the interaction with confounding variables, such as precipitation, solar radiation, humidity, air pressure, atmospheric $\mathrm{CO}_{2}$ concentration, and water and nutrient stresses. Because statistical approaches do not directly consider those factors, results based on statistical approaches only provide suggestive causal relationships between temperature and crop yield and cannot explain processes by which temperature affects crop yield. One important difference from crop simulation models is that, as Liu et al. (2016) pointed out, statistical models "may include indirect effects of climatic variability, such as those related to pests and diseases, which are not well captured by simulation models" (p. 1130).

\section{Conclusions}

Using a regression model and a provincial-scale dataset of observed winter wheat yield and weather variables in China from 1979 to 2011, we investigate the effects of temperature variations on winter wheat yield. To increase the precision of estimated temperature effects and account for the simultaneous variations in weather variables, our regression model includes precipitation and radiation as additional weather variables and considers various region and time fixed effects. In contrast to the existing studies, we discover that winter wheat yield in China responded positively to higher $T_{\min }$, with the positive yield responses varying across wheat growing seasons and winter wheat producing regions. In line with previous assessments, we show that winter wheat yield in China was negatively correlated with higher $T_{\max }$.

Because of the opposing effects of $T_{\min }$ and $T_{\max }$ on winter wheat yield, the estimated net economic impact on China's winter wheat sector stemming from elevated temperatures during the past three decades is sensitive 
to how weather variables are constructed and how the winter wheat growing cycles are defined. Winter wheat yield is projected to decrease modestly by $2.0 \%-7.0 \%$ by 2050 . Our findings of the positive $T_{\min }$ effects and the negative $T_{\max }$ effects on winter wheat yield provide useful information for the development of wheatbreeding programs and the design of efficient adaptation strategies in China to cope with future higher $T_{\max }$, especially when winter wheat is close to maturity in the spring.

Two caveats apply. First, the primary caveat of this work is that estimated weather effects on winter wheat yield are based on an aggregated provincial-scale dataset. Because there exists considerable heterogeneity in crop yields across counties within a province, the estimated effects of weather reported here may deviate from the actual effects of weather on yield. Using a county-level dataset on rice yield, Chen et al. (2016a) analyzed the temperature effect on rice yield in China. The main findings reported in Chen et al. (2016a) hold when a coarse province-level dataset is used (Chen and Tian 2016). Therefore, the main findings reported in this paper might still hold if the temperature effects on winter wheat yield were estimated using a lessaggregated dataset. Second, it is well known that snow cover during the winter months has a positive effect on winter wheat yield because it protects the young wheat crop from fluctuations in ambient temperatures and provides soil moisture required for plant growth in the spring. However, our weather data do not contain information on the presence of snow, thus preventing us from examining the possible effects of snow on winter wheat yield.

Acknowledgments. This paper was supported by the National Natural Science Foundation of China under Grants 71822302 and 71673224. An earlier version of the paper was posted online as a working paper by the Resources for the Future (RFF) and as a discussion paper by the Environment for Development (EfD).

\section{REFERENCES}

Albers, H., C. Gornott, and S. Hüttel, 2017: How do inputs and weather drive wheat yield volatility? The example of Germany. Food Policy, 70, 50-61, https://doi.org/10.1016/ j.foodpol.2017.05.001.

Cameron, A. C., J. B. Gelbach, and D. L. Miller, 2011: Robust inference with multiway clustering. J. Bus. Econ. Stat., 29, 238249, https://doi.org/10.1198/jbes.2010.07136.

Chen, J., Y. Tian, X. Zhang, C. Zheng, Z. Song, A. Deng, and W. Zhang, 2014: Nighttime warming will increase winter wheat yield through improving plant development and grain growth in North China. J. Plant Growth Regul., 33, 397-407, https://doi.org/10.1007/s00344-013-9390-0.
Chen, S., X. Chen, and J. Xu, 2016a: Assessing the impacts of temperature variations on rice yield in China. Climatic Change, 138, 191-205, https://doi.org/10.1007/s10584-016-1707-0.

,-- , and $-2016 \mathrm{~b}$ : Impacts of climate change on agriculture: Evidence from China. J. Environ. Econ. Manage., 76, 105-124, https://doi.org/10.1016/j.jeem.2015.01.005.

Chen, X., and G. Tian, 2016: Impacts of weather variations on rice yields in China based on province-level data. Reg. Environ. Change, 16, 2155-2162, https://doi.org/10.1007/s10113-0160952-0.

, and S. Chen, 2018: China feels the heat: Negative impacts of high temperatures on China's rice sector. Aust. J. Agric. Resour. Econ., 62, 576-588, https://doi.org/10.1111/14678489.12267.

Easterling, D. R., and Coauthors, 1997: Maximum and minimum temperature trends for the globe. Science, 277, 364-367, https://doi.org/10.1126/science.277.5324.364.

Fang, S., K. Tan, S. Ren, X. Zhang, and J. Zhao, 2012: Fields experiments in North China show no decrease in winter wheat yields with night temperature increased by $2.0-2.5^{\circ} \mathrm{C}$. Sci. China Earth Sci., 55, 1021-1027, https://doi.org/10.1007/ s11430-012-4404-5.

_- D. Cammarano, G. Zhou, K. Tan, and S. Ren, 2015: Effects of increased day and night temperature with supplemental infrared heating on winter wheat growth in North China. Eur. J. Agron., 64, 67-77, https://doi.org/10.1016/ j.eja.2014.12.012.

Gibson, L. R., and G. M. Paulsen, 1999: Yield components of wheat grown under high temperature stress during reproductive growth. Crop Sci., 39, 1841-1846, https://doi.org/ 10.2135/cropsci1999.3961841x.

Gourdji, S. M., A. M. Sibley, and D. B. Lobell, 2013: Global crop exposure to critical high temperatures in the reproductive period: Historical trends and future projections. Environ. Res. Lett., 8, 024041, https://doi.org/10.1088/1748-9326/8/2/024041.

Huang, J., C. Xiang, and Y. Wang, 2015: The impact of CIMMYT wheat germplasm on wheat productivity in China. CGIAR Research Program on Wheat Rep., 24 pp., https://repository.cimmyt.org/bitstream/handle/10883/4227/ 99500.pdf? sequence $=1$ \& is Allowed $=y$.

Li, S., T. Wheeler, A. Challinor, E. Lin, H. Ju, and Y. Xu, 2010: The observed relationships between wheat and climate in China. Agric. For. Meteor., 150, 1412-1419, https://doi.org/10.1016/ j.agrformet.2010.07.003.

Liu, B., and Coauthors, 2016: Similar estimates of temperature impacts on global wheat yield by three independent methods. Nat. Climate Change, 6, 1130-1136, https://doi.org/10.1038/ nclimate3115.

Lobell, D. B., and C. B. Field, 2007: Global scale climate-crop yield relationships and the impacts of recent warming. Environ. Res. Lett., 2, 014002, https://doi.org/10.1088/17489326/2/1/014002.

—_, and J. I. Ortiz-Monasterio, 2007: Impacts of day versus night temperatures on spring wheat yields: A comparison of empirical and CERES model predictions in three locations. Agron. J., 99, 469-477, https://doi.org/10.2134/agronj2006.0209.

, , G. P. Asner, P. A. Matson, R. L. Naylor, and W. P. Falcon, 2005: Analysis of wheat yield and climatic trends in Mexico. Field Crops Res., 94, 250-256, https://doi.org/10.1016/ j.fcr.2005.01.007.

, A. Sibley, and J. I. Ortiz-Monasterio, 2012: Extreme heat effects on wheat senescence in India. Nat. Climate Change, 2 , 186-189, https://doi.org/10.1038/nclimate1356. 
Nicholls, N., 1997: Increased Australian wheat yield due to recent climate trends. Nature, 387, 484-485, https://doi.org/10.1038/ $387484 \mathrm{a} 0$.

Peng, S., and Coauthors, 2004: Rice yields decline with higher night temperature from global warming. Proc. Natl. Acad. Sci. USA, 101, 9971-9975, https://doi.org/10.1073/pnas.0403720101.

, and Coauthors, 2013: Asymmetric effects of daytime and night-time warming on Northern Hemisphere vegetation. Nature, 501, 88-92, https://doi.org/10.1038/nature12434.

Porter, J. R., and M. Gawith, 1999: Temperatures and the growth and development of wheat: A review. Eur. J. Agron., 10, 23-36, https://doi.org/10.1016/S1161-0301(98)00047-1.

Prasad, P. V. V., S. R. Pisipati, Z. Ristic, U. Bukovnik, and A. K. Fritz, 2008: Impact of nighttime temperature on physiology and growth of spring wheat. Crop Sci., 48, 2372-2380, https:// doi.org/10.2135/cropsci2007.12.0717.

Tack, J., A. Barkley, and L. L. Nalley, 2015: Effect of warming temperatures on US wheat yields. Proc. Natl. Acad. Sci. USA, 112, 6931-6936, https://doi.org/10.1073/pnas.1415181112.

Tao, F., M. Yokozawa, J. Liu, and Z. Zhang, 2008: Climate-crop yield relationships at provincial scales in China and the impacts of recent climate trends. Climate Res., 38, 83-94, https:// doi.org/10.3354/cr00771.

, D. Xiao, S. Zhang, Z. Zhang, and R. P. Rötter, 2017: Wheat yield benefited from increases in minimum temperature in the Huang-Huai-Hai Plain of China in the past three decades. Agric. For. Meteor., 239, 1-14, https://doi.org/10.1016/ j.agrformet.2017.02.033.

Tashiro, T., and I. Wardlaw, 1989: A comparison of the effect of high temperature on grain development in wheat and rice. Ann. Bot., 64, 59-65, https://doi.org/10.1093/oxfordjournals.aob.a087808.
Warszawski, L., K. Frieler, V. Huber, F. Piontek, O. Serdeczny, and J. Schewe, 2014: The Inter-Sectoral Impact Model Intercomparison Project (ISI-MIP): Project framework. Proc. Natl. Acad. Sci. USA, 111, 3228-3232, https://doi.org/10.1073/ pnas.1312330110.

Welch, J. R., J. R. Vincent, M. Auffhammer, P. F. Moya, A. Dobermann, and D. Dawe, 2010: Rice yields in tropical/subtropical Asia exhibit large but opposing sensitivities to minimum and maximum temperatures. Proc. Natl. Acad. Sci. USA, 107, 14562 14 567, https://doi.org/10.1073/pnas.1001222107.

Xiong, W., I. P. Holman, L. You, J. Yang, and W. Wu, 2014: Impacts of observed growing-season warming trends since 1980 on crop yields in China. Reg. Environ. Change, 14, 7-16, https://doi.org/10.1007/s10113-013-0418-6.

You, L., M. W. Rosegrant, S. Wood, and D. Sun, 2009: Impact of growing season temperature on wheat productivity in China. Agric. For. Meteor., 149, 1009-1014, https://doi.org/10.1016/ j.agrformet.2008.12.004.

Zampieri, M., A. Ceglar, F. Dentener, and A. Toreti, 2017: Wheat yield loss attributable to heat waves, drought and water excess at the global, national and subnational scales. Environ. Res. Lett., 12, 064008, https://doi.org/10.1088/17489326/aa723b.

Zhang, Z., X. Song, F. Tao, S. Zhang, and W. Shi, 2016: Climate trends and crop production in China at county scale, 1980 to 2008. Theor. Appl. Climatol., 123, 291-302, https://doi.org/ 10.1007/s00704-014-1343-4.

Zheng, C., and Coauthors, 2017: Nighttime warming increases winter-sown wheat yield across major Chinese cropping regions. Field Crops Res., 214, 202-210, https://doi.org/10.1016/ j.fcr.2017.09.014. 